\title{
A model for surface diffusion of trans-membrane proteins on lipid bilayers
}

\author{
Ashutosh Agrawal and David J. Steigmann
}

\begin{abstract}
The equilibrium theory of lipid membranes is modified to include the effects of a continuous distribution of trans-membrane proteins. These influence membrane shape and evolve in accordance with a diffusive balance law. The model is purely mechanical in the absence of the proteins. Conditions ensuring energy dissipation in the presence of diffusion are given and an example constitutive function is used to simulate the coupled inertia-less interplay between membrane shape and protein distribution. The work extends an earlier continuum theory of equilibrium configurations of composite lipid-protein membranes to accommodate surface diffusion.
\end{abstract}

Mathematics Subject Classification (2000). 92B05.

Keywords. Lipid membranes · Protein diffusion.

\section{Introduction}

Lipid bilayers are composed of transversely oriented lipid molecules containing hydrophilic head groups and hydrophobic tails [1]. These groups are arranged in opposing orientations that shield the tail groups from the surrounding aqueous solution. Relative misalignment of the lipids entails an energetic cost which manifests itself as flexural stiffness of the film, while the bilayer is otherwise fluid-like in its response. Further, bilayers are naturally symmetric with respect to a midsurface and so lack a natural orientation. Kim et al. [2] have demonstrated that proteins play a principal role in the morphology of lipid bilayers in which they are embedded. In particular, it has been shown that transmembrane proteins which are roughly conical in shape, with the vertex lying to one side of the membrane, induce natural membrane bending as a consequence of local protein-lipid interactions. These interactions effectively break the bilayer symmetry, producing a natural curvature of the membrane over patches of surface containing many similar proteins.

The influence of transmembrane proteins on membrane shape has been studied in $[3,4]$, for example. In particular, [3] is closely related to the work described here. However, being based on linear geometric approximations in the Monge representation of a surface, it is limited in scope to small deviations of membrane shape from a plane. The present work does not entail such restrictions and is thus applicable to general membrane geometries. Further, surface tension is suppressed in [3]. Here we find the effects of surface tension and its gradient, induced by variations in protein concentration over the surface, to be non-negligible and to persist in the geometrically linear limit considered in [3]. Beyond these generalizations, we find it necessary to account explicitly for membrane curvature as well as tangential and normal membrane velocities in our diffusive balance law. These effects are also suppressed in [3,4], although our constitutive equation for protein flux, based on a chemical potential for protein concentration, is similar in principle to that adopted in [3]. Thus, the present work may be viewed as a nonlinear extension and generalization of [3]. Unlike [3], however, we do not incorporate viscous interaction between the membrane and the aqueous solution in which it is immersed. To model bulk interactions, some workers adopt a firstorder-in-time model for shape evolution of the membrane [4]. This is a non-local equation incorporating bulk viscosity and which requires membrane shape to evolve in response to the variational derivative of 
the total membrane bending energy. This model is based on the Green's function for Stokes flow in the bulk [5]. As in [3], its use has been confined to the linearized Monge representation for membranes [4]. Further, it does not account for intra-membrane viscous flow; that is, flow of lipids in the membrane surface. However, in [6], the relative importance of intra-membrane viscosity vis a vis bulk viscosity is demonstrated at biologically relevant length scales on the basis of a nonlinear model in which membrane geometry is taken fully into account. The present study, concerned with shape evolution coupled to protein diffusion in isolation from such effects, should be viewed simply as supplying one ingredient of a general model that accommodates diffusion as well as extra- and intra-membrane viscous interactions. Thus, we confine attention to the effects of protein diffusion per se. In this respect, our objectives are the same as those addressed in [7].

We discuss a framework for bilayer membranes in which it is assumed that trans-membrane proteins are continuously distributed. Their associated areal concentration is assumed to evolve in a diffusive manner over the composite lipid-protein surface. The natural curvature induced by proteins on the local scale is modeled in terms of the dependence of the spontaneous curvature on protein concentration, in accordance with our earlier hypothesis [8] and that of the originators of the concept [3]. In contrast, spontaneous curvature is regarded as a fixed parameter in the conventional theory of membrane equilibria $[1,9]$. A plausible assumption, adopted here, is that spontaneous curvature is proportional to protein concentration, with the sign of the constant of proportionality being that of the natural curvature induced by local protein geometry.

Section 2 contains a summary of the basic equations of the standard model, including the requisite differential geometry, the kinematics of surface deformation, and the equilibrium equations pertaining to inertia-less motions. These matters are discussed more comprehensively elsewhere [10,11]. A balance law for surface diffusion is discussed in Sect. 3. This makes explicit the interplay between concentration, membrane geometry, and the normal and tangential components of surface velocity. In Sect. 4, we propose an extension of the standard equilibrium membrane energy to account for protein concentration and combine this with the diffusive balance law to derive a condition ensuring that protein diffusion is a dissipative process. A simple constitutive equation is proposed for the associated concentration flux which accommodates classical Fickean diffusion in addition to non-standard effects associated with membrane curvature.

Finally, in Sect. 5, we specialize the model to the case of axisymmetry and, in Sect. 6, illustrate it through numerical solution of the associated differential equations.

\section{Surface geometry, the description of motion and mechanical equilibrium}

We denote the membrane surface by $\omega$. Position on this surface, relative to a specified origin, is described in parametric form by the function $\mathbf{r}\left(\theta^{\mu}, t\right)$, where $\theta^{\mu} ; \mu=1,2$, are surface coordinates. These coordinates play a role analogous to that played by a fixed-coordinate system used to parametrize a control volume in the Eulerian or spatial description of classical fluid mechanics. Here, and henceforth, Greek indices range over $\{1,2\}$ and, if repeated, are summed over that range. We assume familiarity with tensor analysis and curvilinear coordinate systems. Useful introductions for mechanicians are given in [12-15]. Subscripts preceded by commas indicate partial derivatives with respect to the coordinates, while those preceded by semicolons indicate covariant derivatives. The surface coordinates induce the basis $\mathbf{a}_{\alpha}=\mathbf{r}_{, \alpha}$ for the tangent plane to $\omega$ at the point with coordinates $\theta^{\mu}$. The induced metric is $a_{\alpha \beta}=\mathbf{a}_{\alpha} \cdot \mathbf{a}_{\beta}$, and is assumed to be positive definite. A dual basis on the tangent plane is then given by $\mathbf{a}^{\alpha}=a^{\alpha \beta} \mathbf{a}_{\beta}$, where $\left(a^{\alpha \beta}\right)=\left(a_{\alpha \beta}\right)^{-1}$. The local orientation of the surface is defined by the unit-normal field

$$
\mathbf{n}=\mathbf{a}_{1} \times \mathbf{a}_{2} /\left|\mathbf{a}_{1} \times \mathbf{a}_{2}\right|
$$


and its local curvature by the surface-tensor field

$$
\mathbf{b}=b_{\alpha \beta} \mathbf{a}^{\alpha} \otimes \mathbf{a}^{\beta},
$$

where

$$
b_{\alpha \beta}=\mathbf{n} \cdot \mathbf{r}_{, \alpha \beta}=-\mathbf{a}_{\alpha} \cdot \mathbf{n}_{, \beta} .
$$

If $\boldsymbol{\nu}$ and $\mathbf{t}$ are orthonormal vectors in the tangent plane, then

$$
\mathbf{b}=\kappa_{\nu} \boldsymbol{\nu} \otimes \boldsymbol{\nu}+\kappa_{t} \mathbf{t} \otimes \mathbf{t}+\tau(\boldsymbol{\nu} \otimes \mathbf{t}+\mathbf{t} \otimes \boldsymbol{\nu}),
$$

where $\kappa_{\nu}$ and $\kappa_{t}$ are the normal curvatures on these axes and $\tau$ is the twist. The mean and Gaussian curvatures are

$$
H=\frac{1}{2}\left(\kappa_{\nu}+\kappa_{t}\right) \text { and } K=\kappa_{\nu} \kappa_{t}-\tau^{2},
$$

respectively.

To ease the formulation of balance laws, we introduce a convected coordinate system $\xi^{\alpha}$. These may be identified with the $\theta^{\alpha}$ at a fixed instant $t_{0}$. The associated surface $\Omega$, with parametric representation $\mathbf{x}\left(\xi^{\alpha}\right)=\mathbf{r}\left(\xi^{\alpha}, t_{0}\right)$, is fixed and may serve as a reference surface in a Lagrangian or referential description of the motion. That is, we regard these coordinates as being convected in the sense that they identify, via a map $\mathbf{r}=\hat{\mathbf{r}}\left(\xi^{\alpha}, t\right)$, the current position at time $t$ of a point that was located at $\mathbf{x}\left(\xi^{\alpha}\right) \in \Omega$ at time $t_{0}$. The connection with the $\theta^{\alpha}$-parametrization of $\omega$ is provided by $[12,16]$

$$
\hat{\mathbf{r}}\left(\xi^{\alpha}, t\right)=\mathbf{r}\left(\theta^{\alpha}\left(\xi^{\beta}, t\right), t\right) .
$$

Thus, we specify the fixed surface coordinates $\theta^{\alpha}$ as functions of $\xi^{\alpha}$ and $t$ subject to the initial condition $\theta^{\alpha}\left(\xi^{\beta}, t_{0}\right)=\xi^{\alpha}$. More will be said about this relationship, but first we note that once it is specified, any function, $f\left(\theta^{\alpha}, t\right)$, say, may be expressed in terms of convected coordinates as $\hat{f}\left(\xi^{\alpha}, t\right)$, where

$$
\hat{f}\left(\xi^{\alpha}, t\right)=f\left(\theta^{\alpha}\left(\xi^{\beta}, t\right), t\right) .
$$

The time derivative of a function in the convected coordinate representation is $\dot{f}=\partial \hat{f}\left(\xi^{\alpha}, t\right) / \partial t$, whereas that in the fixed-coordinate parametrization is $f_{t}=\partial f\left(\theta^{\alpha}, t\right) / \partial t$. By the chain rule, the two are related by $\dot{f}=f_{t}+\left(\theta^{\alpha}\right)^{\cdot} f_{, \alpha}$.

The velocity of a point on $\Omega$ that has been convected by the motion to $\omega$ is $\mathbf{u}=\dot{\mathbf{r}}=\partial \hat{\mathbf{r}} / \partial t$. We may write this in terms of components on the natural basis induced by the fixed-coordinate $\left(\theta^{\alpha}\right)$ parametrization. Thus,

$$
\mathbf{u}=u^{\alpha} \mathbf{a}_{\alpha}+u \mathbf{n} .
$$

This is not the same as the time derivative $\mathbf{r}_{t}$. The two are related by

$$
\mathbf{u}=\left(\theta^{\alpha}\right) \cdot \mathbf{a}_{\alpha}+\mathbf{r}_{t} .
$$

Following $[12,16]$, we adopt the fixed-coordinate parametrization defined by

$$
\frac{\mathrm{d}}{\mathrm{d} t} \theta^{\alpha}=u^{\alpha}\left(\theta^{\beta}, t\right), \quad \theta_{\mid t_{0}}^{\alpha}=\xi^{\alpha},
$$

where the derivative is evaluated at a fixed value of the doublet $\left\{\xi^{\alpha}\right\}$ and is therefore equal to $\left(\theta^{\alpha}\right)$. Accordingly, the normal velocity in (8) is given by

$$
u \mathbf{n}=\mathbf{r}_{t},
$$

and the convected and fixed-coordinate time derivatives satisfy

$$
\dot{f}=f_{t}+u^{\alpha} f_{, \alpha} .
$$

Alternatively, it is sometimes convenient to regard the $\theta^{\alpha}$ as being convected, especially in problems involving configurations that do not evolve in time [17]. In particular, this choice affords some simplification in the computation of the Gateaux derivatives of the membrane energy [18]. 
The foregoing relationships facilitate the reduction of global balance laws. For example, if $f$ is the areal density of a particular quantity on $\omega$, then the rate of change of the total quantity in a part $\pi$ of $\omega$ is

$$
\frac{\mathrm{d}}{\mathrm{d} t} \int_{\pi} f \mathrm{~d} a=\frac{\mathrm{d}}{\mathrm{d} t} \int_{\Pi} f J \mathrm{~d} A=\int_{\pi}(\dot{f}+f \dot{J} / J) \mathrm{d} a,
$$

where $\Pi$ is the part of the fixed surface $\Omega$ that is convected to $\pi$ and $J$ is the local areal dilation of the surface; i.e.,

$$
\int_{\pi} \mathrm{d} a=\int_{\Pi} J \mathrm{~d} A \text { for all } \Pi \subset \Omega .
$$

To express the right-hand side of (13) in terms of the fixed-coordinate parametrization, we use [18]

$$
\dot{J} / J=u_{; \alpha}^{\alpha}-2 H u \text {. }
$$

Suppose the membrane is incompressible in the two-dimensional sense; i.e., $J=1$. The constitutive response of conventional lipid membranes is then described by a function $W\left(H, K ; \theta^{\alpha}\right)$, the bending energy per unit area of $\omega$. If the membrane is in mechanical equilibrium under a net lateral pressure $p$ in the direction of its orientation $\mathbf{n}$, then the shape equation $[1,10,11]$

$$
p=\Delta\left(\frac{1}{2} W_{H}\right)+\left(W_{K}\right)_{; \alpha \beta} \tilde{b}^{\alpha \beta}+W_{H}\left(2 H^{2}-K\right)+2 H\left(K W_{K}-W\right)-2 \lambda H
$$

is operative, where $\Delta(\cdot)=(\cdot)_{; \alpha \beta} a^{\alpha \beta}$ is the surface Laplacian, and

$$
\tilde{b}^{\alpha \beta}=2 H a^{\alpha \beta}-b^{\alpha \beta}
$$

is the cofactor of the curvature. The scalar field $\lambda$ is a Lagrange multiplier associated with the incompressibility constraint. This satisfies [8]

$$
\lambda_{, \alpha}=-\partial W / \partial \theta^{\alpha}
$$

in which the right-hand side is the derivative of the function $W(H, K ; \cdot)$. This derivative accounts for any explicit coordinate dependence in the bending energy, whereas implicit dependence occurs via the coordinate dependence of $H$ and $K$. Said differently, the right-hand side of (18) accommodates any non-uniformity in the bending properties of the membrane. This feature plays a central role in the present work, in which such non-uniformity is generated by the constitutive response of the membrane to transmembrane proteins.

\section{Surface diffusion}

The transport of proteins on the membrane surface is assumed to occur by diffusion. Thus, if $\sigma\left(\theta^{\alpha}, t\right)$ is the areal concentration of proteins (number of proteins of a given type per unit current membrane area), then for any simply connected part $\pi$ of the current membrane surface $\omega$, we have

$$
\frac{\mathrm{d}}{\mathrm{d} t} \int_{\pi} \sigma \mathrm{d} a=-\int_{\partial \pi} \mathbf{m} \cdot \boldsymbol{\nu} \mathrm{d} s, \quad \text { all } \quad \pi \subset \omega,
$$

where the surface vector $\mathbf{m}=m^{\alpha} \mathbf{a}_{\alpha}$ is the protein flux. Here and elsewhere, $\boldsymbol{\nu}=\nu^{\alpha} \mathbf{a}_{\alpha}$ is the exterior unit normal to the edge $\partial \pi$, defined by $\boldsymbol{\nu}=\mathbf{t} \times \mathbf{n}$, where $\mathbf{t}=\mathbf{a}_{\alpha}\left(\mathrm{d} \theta^{\alpha} / \mathrm{d} s\right)$ and $\theta^{\alpha}(s)$ is the arclength parametrization of $\partial \pi$ at time $t$. We assume that there is no transfer of proteins between the membrane and the bulk fluid in which it is immersed. Such transfer would require the addition of an areal source term to (19). 
To extract a local equation from (19), we use the transport Eq. (13) together with Stokes' theorem, obtaining

$$
\int_{\pi}(\dot{\sigma}+\sigma \dot{J} / J) \mathrm{d} a=-\int_{\pi} m_{; \alpha}^{\alpha} \mathrm{d} a
$$

where

$$
m_{; \alpha}^{\alpha}=a^{-1 / 2}\left(a^{1 / 2} m^{\alpha}\right)_{, \alpha},
$$

in which $a=\operatorname{det}\left(a_{\alpha \beta}\right)$, is the surface divergence of $\mathbf{m}$. Thus, from (12), (15), and the localization theorem,

$$
\sigma_{t}+u^{\alpha} \sigma_{, \alpha}+\sigma\left(u_{; \alpha}^{\alpha}-2 H u\right)+m_{; \alpha}^{\alpha}=0 \quad \text { on } \quad \omega .
$$

If the membrane is incompressible in the two-dimensional sense, then $J=1$ and $\dot{J}=0$, and the velocity field and protein concentration satisfy

$$
u_{; \alpha}^{\alpha}=2 H u \quad \text { and } \quad \sigma_{t}+u^{\alpha} \sigma_{, \alpha}+m_{; \alpha}^{\alpha}=0 \quad \text { on } \quad \omega .
$$

\section{Energy and its dissipation by diffusion}

In [8], we assumed that protein density affects membrane shape via the so-called spontaneous curvature [9], thus accommodating earlier ideas about protein-membrane interactions within a continuum-based framework (see also [3]). This amounts to a particular kind of parametric dependence of membrane energy on protein density. Generalizing this idea, we consider membrane energies of the form $\tilde{W}(J, H, K, \sigma)$. The connection with the conventional bending energy used in (16), at time $\bar{t}$, say, is

$$
W\left(H, K ; \theta^{\alpha}\right)=\tilde{W}\left(1, H, K, \sigma\left(\theta^{\alpha}, \bar{t}\right)\right) .
$$

Thus, any explicit coordinate dependence in the function of $H$ and $K$ defined by $W\left(\cdot, \cdot ; \theta^{\alpha}\right)$ occurs through $\sigma$, and the conventional coordinate-dependent bending energy function may be used in the treatment of equilibrium problems provided that it evolves in time in response to the transmembrane proteins, in accordance with (24).

The importance of the connection (24) to the present work is due to our restriction to processes occurring so slowly that the inertia of the composite lipid-protein membrane may be neglected. The composite membrane is thus deemed to be in mechanical equilibrium at every instant in the course of such a process. This implies that (16) is effective at each instant, with $W$ given by (24). Accordingly, the only contribution to the energy of the system is that stored in the composite membrane. This energy changes in response to the forces and couples applied to the membrane and to the flux of proteins across its boundary. However, because diffusion is a dissipative process, there is a discrepancy between the rate at which energy is supplied and that at which it is stored. This dissipation, denoted by $D$, is given by

$$
D(\pi, t)=P(\pi, t)-\frac{\mathrm{d}}{\mathrm{d} t} E(\pi, t),
$$

where $\pi$ is any simply connected part of $\omega$,

$$
E(\pi, t)=\int_{\pi} \tilde{W}(J, H, K ; \sigma) \mathrm{d} a
$$

is the membrane energy, and, for smooth boundary $\partial \pi$,

$$
P(\pi, t)=\int_{\pi} p \mathbf{n} \cdot \mathbf{u} \mathrm{d} a+\int_{\partial \pi} \mathbf{F} \cdot \mathbf{u} \mathrm{d} s-\int_{\partial \pi} M \mathbf{t} \cdot \boldsymbol{\omega} \mathrm{d} s-\int_{\partial \pi} \mathbf{q} \cdot \boldsymbol{\nu} \mathrm{d} s
$$

is the energy supply rate, in which $p$ is the net pressure on the membrane, $\mathbf{F}$ is the applied force per unit length, $-M$ is the applied bending moment per unit length (the sign conforming to the convention 
adopted in [17]), $\boldsymbol{\omega}$ is the rate of change of membrane orientation (i.e., $\mathbf{\mathbf { n }}=\boldsymbol{\omega} \times \mathbf{n}$ ) and $\mathbf{q}$ is the rate at which membrane energy changes due to protein flux. Constitutive equations for $\mathbf{F}$ and $M$ in terms of membrane properties are given in [17]. The term $\mathbf{q}$ represents the rate of change of the energy of the composite lipid-membrane surface due to the flux of protein density. Accordingly, it is given by

$$
\mathbf{q}=W_{\sigma} \mathbf{m}
$$

in which the constitutive equation for $\mathbf{m}$ has yet to be specified. Thus, $W_{\sigma}$ is the chemical potential for the diffusing proteins. This result also follows as a necessary condition in a rigorous thermodynamic treatment of dissipation associated with diffusive processes [19].

We assume that

$$
D(\pi, t) \geq 0,
$$

this effectively serving as the second law of thermodynamics in the present setting.

The well-known Helfrich model [9] applies if the membrane curvature, non-dimensionalized by bilayer thickness (on the order of molecular dimensions), is small. In this case, a quadratic dependence on (dimensionless) curvature is often sufficient and will be assumed here. Thus,

$$
\tilde{W}(J, H, K, \sigma)=a(J, \sigma)+b(J, \sigma) H+c(J, \sigma) H^{2}+d(J, \sigma) K
$$

where $a-d$ are suitable functions. If the dimensionless curvature is negligible, as in soap films of macroscopic size, then this yields $W \simeq a(J, \sigma)$, furnishing a generalization of classical capillarity theory to accommodate both surface elasticity $[20,21]$ and diffusion. The present work, however, is concerned with conditions in which the energetic cost of areal dilation is significant compared to that associated with bending, as in the conventional theory of lipid membranes [1]. This situation is prevalent in cell biomechanics [22]. We further suppose the protein density to be small enough that areal dilation is accommodated primarily by the lipids and thus negligible. Accordingly, we suppress dilation in the ensuing development. Its effects may be included at the modeling level by a minor adjustment to our procedure. Thus, we impose $J=1$ in (30). We further suppose that there exists a value, $C$, of mean curvature-the spontaneous curvature - at which $W_{H}$ vanishes. This leads to the special case of (30) defined by $\tilde{W}=\bar{W}$, where

$$
\bar{W}(H, K, \sigma)=A(\sigma)+k(\sigma)[H-C(\sigma)]^{2}+\bar{k}(\sigma) K,
$$

which furnishes a natural extension of the Helfrich energy to accommodate protein density. We suppose that the membrane possesses bilayer symmetry in the absence of transmembrane proteins (i.e., $\bar{W}(\cdot, K, 0)$ an even function), and thus require that $C(0)=0$. Henceforth, to ease the notation, we use the symbol $W$ to denote the membrane energy defined by (31).

With the constraint in force, the membrane energy

$$
E=\int_{\pi} W \mathrm{~d} a
$$

is equal, in all admissible states of the membrane, to

$$
E^{\prime}=\int_{\Pi}[J W+\lambda(J-1)] \mathrm{d} A,
$$

where $\lambda$ is the Lagrange multiplier field $[8,17]$. Accordingly, $\mathrm{d} E / \mathrm{d} t=\mathrm{d} E^{\prime} / \mathrm{d} t$, where

$$
\frac{\mathrm{d}}{\mathrm{d} t} E^{\prime}=\int_{\pi}\left[\dot{W}+(W+\lambda) \dot{J} / J+\dot{\lambda}\left(1-J^{-1}\right)\right] \mathrm{d} a,
$$

in which

$$
\dot{W}=W_{H} \dot{H}+W_{K} \dot{K}+W_{\sigma} \dot{\sigma}
$$


If the membrane is in mechanical equilibrium, as assumed here, then the virtual-work principle yields [17]

$$
\frac{\mathrm{d}}{\mathrm{d} t} E_{\mid \sigma}^{\prime}=\int_{\pi} p \mathbf{n} \cdot \mathbf{u} \mathrm{d} a+\int_{\partial \pi} \mathbf{F} \cdot \mathbf{u} \mathrm{d} s-\int_{\partial \pi} M \mathbf{t} \cdot \boldsymbol{\omega} \mathrm{d} s,
$$

where the subscript refers to the derivative evaluated at fixed $\sigma$; i.e., with $t$ fixed in the function $\hat{\sigma}\left(\xi^{\alpha}, t\right)$. That is, Eqs. (16) and (18), together with appropriate side conditions, satisfy (36) at fixed $\sigma$ [17], provided that the Lagrange multiplier $\lambda$ satisfies [cf. (18) and (24)]

$$
\nabla \lambda=-W_{\sigma} \nabla \sigma
$$

Using (36), Eq. (25) then reduces to

$$
D(\pi, t)=-\int_{\partial \pi} W_{\sigma} \mathbf{m} \cdot \boldsymbol{\nu} \mathrm{d} s-\int_{\pi} W_{\sigma} \dot{\sigma} \mathrm{d} a .
$$

We substitute $(23)_{2}$ in the form $\dot{\sigma}=-m_{; \alpha}^{\alpha}$ and apply Stokes' theorem to derive

$$
D(\pi, t)=-\int_{\pi} \mathbf{m} \cdot \nabla\left(W_{\sigma}\right) \mathrm{d} a .
$$

Inequality (29) and the arbitrariness of $\pi$ lead finally to the restriction

$$
\mathbf{m} \cdot \nabla\left(W_{\sigma}\right) \leq 0 .
$$

Here, $\mathbf{m}$ is assumed to depend constitutively on $\nabla\left(W_{\sigma}\right)$. Following a standard argument [23], (40) may then be used to show that $\mathbf{m}$ vanishes if $\nabla\left(W_{\sigma}\right)$ vanishes. The simplest such model is $\mathbf{m}=-c \nabla\left(W_{\sigma}\right)$, where $c$ is a constant; i.e.,

$$
\mathbf{m}=-c \nabla\left(W_{\sigma}\right)
$$

Inequality (40), the left-hand side of which reduces to $-c\left|\nabla\left(W_{\sigma}\right)\right|^{2}$, then requires that $c \geq 0$. This model incorporates classical Fickean diffusion. To see this, we suppose $k, \bar{k}$ and $C$ to be constants in (31), obtaining $W_{\sigma}=A^{\prime}(\sigma)$ and $\nabla\left(W_{\sigma}\right)=A^{\prime \prime}(\sigma) \nabla \sigma$. Fick's law is recovered on specializing $A^{\prime \prime}(\sigma)$ to be a positive constant. In a related work [7], protein flux is assumed to be directly proportional to the gradient of concentration rather than gradient of chemical potential, while the dependence of spontaneous curvature on concentration is retained. This is not consistent with the present framework and so it is not possible to conclude that diffusion is dissipative in the context of the model adopted in [7].

Proceeding with a model of the influence of protein density on membrane shape, we adopt the simplest generalization of our earlier proposal [8]. Thus, we assume that

$$
W=A(\sigma)+k[H-C(\sigma)]^{2} .
$$

This corresponds to the restriction of (31) to the case of constant bending moduli $k$ and $\bar{k}$, which in turn is justified by our earlier assumption of a dilute concentration of proteins, their effect on bending stiffness thus being negligible. In this case, the term involving $K$ does not appear in the equilibrium Eqs. (16) and (37), and may therefore be suppressed for closed membranes without boundary. However, it does affect boundary conditions involving forces and bending moments [17]. Nevertheless, for the sake of illustration, we suppress it in the examples discussed in Sect. 6.

Roughly, a transmembrane protein may be idealized as being conical in shape, with its axis of revolution directed along the surface normal $\mathbf{n}$. Local hydrophobic lipid-protein interactions thus promote a natural membrane curvature that depends on protein geometry and density [2]. If $\varphi$ is the angle made by the meridian of the cone with $\mathbf{n}$, then it is plausible that this spontaneous curvature is proportional to protein density, and that the constant of proportionality is itself proportional to cone angle. A simple proposal that ensures bilayer symmetry in the absence of proteins is 


$$
C(\sigma)=(\mu \varphi) \sigma
$$

where $\varphi \in(-\pi / 2, \pi / 2)$ is the cone angle and $\mu$ is a positive constant. Thus, we propose

$$
W=(\alpha \sigma-\beta)^{2}+k[H-C(\sigma)]^{2}
$$

in which $\alpha, \beta$ are positive constants, which conforms to the conventional theory of bending elasticity in the absence of diffusion provided that $k>0$ [24]. We have

$$
W_{\sigma}=2[\alpha(\alpha \sigma-\beta)+k \mu \varphi(C-H)],
$$

and the protein flux is

$$
\mathbf{m}=-2 c\left\{\left[\alpha^{2}+k(\mu \varphi)^{2}\right] \nabla \sigma-k \mu \varphi \nabla H\right\}
$$

in which $\nabla C=\mu \varphi \nabla \sigma$.

The equilibrium shape equation corresponding to (44) is

$$
k \Delta(H-C)+2 k(H-C)\left(2 H^{2}-K\right)-2 H\left[(\alpha \sigma-\beta)^{2}+k(H-C)^{2}\right]=p+2 \lambda H,
$$

and Eq. (37) for the Lagrange multiplier reduces to

$$
\nabla \lambda=2[k \mu \varphi(H-C)-\alpha(\alpha \sigma-\beta)] \nabla \sigma .
$$

The constitutive Eq. (46) spans the range from bending-dominated response $(\mu|\varphi| \sqrt{k} \gg \alpha)$ to classical Fickean diffusion $(\mu|\varphi| \sqrt{k} \ll \alpha)$, the latter applying to cylindrical transmembrane proteins $(\varphi=0)$ and macroscopic soap films. In the former case, the important features of the response are captured by taking

$$
W \approx k[H-C(\sigma)]^{2}, \quad W_{\sigma} \approx 2 k \mu \varphi(C-H) \quad \text { and } \quad \mathbf{m} \approx-2 c k \mu \varphi \nabla(C-H) .
$$

We observe in this case that diffusion ceases and the energy is minimized locally (hence globally) if $H=C(\sigma)$, in which $\sigma$ may be non-uniform on the surface. In this case, the shape equation with $p=0$ is satisfied provided that $\lambda=-(\alpha \sigma-\beta)^{2}$. The existence of such a state is contingent on satisfaction of operative boundary data together with the Gauss and Mainardi-Codazzi compatibility conditions of differential geometry [13]. If satisfied, the theory then yields a non-uniformly curved composite lipid-protein configuration that is energetically optimal in the absence of lateral pressure. The solutions reported in [8] are equilibrium states of precisely this kind. In intermediate cases, the infimum of the energy is achieved and diffusion ceases, again in the absence of pressure, if $\lambda=0, \sigma=\sigma_{0}$ and $H=C\left(\sigma_{0}\right)$, where $\sigma_{0}=\beta / \alpha$.

We discuss several illustrative examples in Sect. 6 below, all for the case of zero lateral pressure and with all fields possessing axial symmetry on a surface of revolution.

\section{Surfaces of revolution}

We consider axisymmetric fields on surfaces of revolution parametrized, in all configurations, by meridional arclength $s$ and azimuthal angle $\theta$. Thus,

$$
\mathbf{r}(s, \theta, t)=r(s, t) \mathbf{e}_{r}(\theta)+z(s, t) \mathbf{k}
$$

is the position field on the surface, where $r(s, t)$ is the radius from the axis of symmetry, $z(s, t)$ is the elevation above a base plane, and $\left\{\mathbf{e}_{r}, \mathbf{e}_{\theta}, \mathbf{k}\right\}$ is the standard orthonormal basis in a system of cylindrical polar coordinates $\{r, \theta, z\}$. Meridians and parallels of latitude are the curves on which $\theta$ and $s$, respectively, are constant. Further, because $s$ measures arclength along meridians, we have

$$
\left(r^{\prime}\right)^{2}+\left(z^{\prime}\right)^{2}=1
$$

where $(\cdot)^{\prime}$ is used to denote differentiation with respect to $s$. 
We select surface coordinates $\theta^{1}=s$ and $\theta^{2}=\theta$ and identify the vector $\boldsymbol{\nu}$ of (2) with the unit tangent to a meridian. The induced tangent vectors are

$$
\mathbf{a}_{1}=r^{\prime} \mathbf{e}_{r}+z^{\prime} \mathbf{k} \text { and } \mathbf{a}_{2}=r \mathbf{e}_{\theta},
$$

and it follows from $(51)$ that there is $\psi(s, t)$ such that

$$
r^{\prime}=\cos \psi \quad \text { and } \quad z^{\prime}=\sin \psi
$$

Because $\mathbf{a}_{1}$ is orthogonal to a parallel of latitude, we identify it with $\boldsymbol{\nu}$ in (19), etc. Accordingly,

$$
\boldsymbol{\nu}=\cos \psi \mathbf{e}_{r}+\sin \psi \mathbf{k}, \quad \mathbf{t}=\mathbf{e}_{\theta} \quad \text { and } \quad \mathbf{n}=\cos \psi \mathbf{k}-\sin \psi \mathbf{e}_{r} .
$$

The metric and dual metric are $\left(a_{\alpha \beta}\right)=\operatorname{diag}\left(1, r^{2}\right)$ and $\left(a^{\alpha \beta}\right)=\operatorname{diag}\left(1, r^{-2}\right)$, respectively, and the latter may be used to compute

$$
\mathbf{a}^{1}=\boldsymbol{\nu} \quad \text { and } \quad \mathbf{a}^{2}=r^{-1} \mathbf{e}_{\theta} .
$$

To obtain the components of curvature, we use (3), obtaining $\left(b_{\alpha \beta}\right)=\operatorname{diag}\left(\psi^{\prime}, r \sin \psi\right)[24]$. Combining this with (4) and (54), we derive

$$
\kappa_{\nu}=\psi^{\prime}, \quad \kappa_{t}=r^{-1} \sin \psi \quad \text { and } \quad \tau=0 .
$$

The sum of the normal curvatures is twice the mean curvature $H(s, t)$. This furnishes the differential equation

$$
r \psi^{\prime}=2 r H-\sin \psi .
$$

Their product is the Gaussian curvature $K(s, t)$; thus,

$$
K=H^{2}-\left(H-r^{-1} \sin \psi\right)^{2} .
$$

The equilibrium shape Eq. (47) simplifies, in the absence of lateral pressure, to $[17,24]$

$$
L^{\prime}=2 r\left\{H\left[k(H-C)^{2}+(\alpha \sigma-\beta)^{2}+\lambda\right]-k(H-C)\left[H^{2}+\left(H-r^{-1} \sin \psi\right)^{2}\right]\right\},
$$

where

$$
L=\frac{1}{2} r\left(W_{H}\right)^{\prime}=k r\left(H^{\prime}-\mu \varphi \sigma^{\prime}\right),
$$

and the Lagrange multiplier satisfies the ordinary differential equation

$$
\lambda^{\prime}=2[k \mu \varphi(H-C)-\alpha(\alpha \sigma-\beta)] \sigma^{\prime} .
$$

Equation $(23)_{1}$, relating the normal and tangential velocities of the surface, reduces to

$$
(r v)^{\prime}=2 r H u,
$$

where $v(s, t)$ is the velocity component in the direction of the tangent to the meridian; we assume the velocity in the azimuthal direction to vanish. This furnishes the tangential velocity gradient and thus may be used to estimate the error incurred by neglecting intra-membrane viscosity.

From Eq. (46) for the protein flux, we obtain

$$
m=-c\left(W_{\sigma}\right)^{\prime}=2 c\left[k \mu \varphi H^{\prime}-\left(\alpha^{2}+k \mu^{2} \varphi^{2}\right) \sigma^{\prime}\right],
$$

where $m(=\mathbf{m} \cdot \boldsymbol{\nu})$ is the component of the flux $\mathbf{m}$ in the direction tangent to the meridian; the azimuthal component of this vector also vanishes. Finally, the diffusive balance law $(23)_{2}$ reduces to

$$
\sigma_{t}+v \sigma^{\prime}=\frac{2 c}{r}\left\{r\left[\left(\alpha^{2}+k \mu^{2} \varphi^{2}\right) \sigma^{\prime}-k \mu \varphi H^{\prime}\right]\right\}^{\prime} .
$$

To specify the domain on which the foregoing differential equations are to be solved, we note that the local constraint $\dot{J}=0$ represented by (62) implies, via (14), a global constraint on the area of the current surface. At any fixed time, the surface area of the sector $(0, s)$ is

$$
a(s, t)=2 \pi \int_{0}^{s} r(x, t) \mathrm{d} x .
$$


Accordingly,

$$
a^{\prime}=2 \pi r .
$$

Because this is strictly positive in $(0, s)$, we may use it to effect a change of independent variable from $s$ to $a$, and integrate the resulting system on the domain $(0, A)$, where $A$ is the assigned area of the membrane. The system thus derived is non-dimensionalized by using $R$ as the length scale, where $A=\pi R^{2}$, and using $\hat{\tau}=(c k)^{-1}$ as the time scale, the latter following from a dimensional analysis of the balance law (64) together with the constitutive equation (63). The nondimensional variables are

$$
\begin{aligned}
& \bar{a}=a / R^{2}, \quad \bar{r}=r / R, \quad \bar{z}=z / R, \quad \bar{H}=R H, \quad \bar{C}=R C, \quad \bar{L}=R L / k, \quad \bar{\lambda}=R^{2} \lambda / k, \quad \bar{s}=s / R, \\
& \bar{t}=t / \hat{\tau}, \quad \bar{\alpha}=\alpha /(R \sqrt{k}), \quad \bar{\beta}=\beta R / \sqrt{k}, \quad \bar{\mu}=\mu / R, \quad \bar{u}=u \hat{\tau} / R, \quad \bar{v}=v \hat{\tau} / R \quad \text { and } \quad \bar{\sigma}=R^{2} \sigma .
\end{aligned}
$$

An associated system of dimensionless equations is easily obtained, but for the sake of brevity we do not record it here.

To ensure that numerical solutions yield positive values of protein density, we introduce an auxiliary variable $\tilde{\sigma}$ defined by $\bar{\sigma}=\exp \tilde{\sigma}$. The equations are solved by specifying the distribution of $\bar{\sigma}$ at an initial time $\bar{t}=0$, say, and setting the initial normal and tangential velocities to zero. We then compute the initial membrane shape by integrating Eqs. (59)-(61) using Matlab ('bvp4c'). Equation (64) is used to compute the spatial distribution of the initial value of the time derivative of the protein density. The protein distribution at time $\Delta \bar{t}$ is then obtained from a forward Euler approximation to the time derivative, where $\Delta \bar{t}$ is a suitable time step. The shape at time $\Delta \bar{t}$ is then obtained by integrating (59)-(61), as before. With the shapes known at times zero and $\Delta \bar{t}$, we form a backward Euler approximation, at each mesh point, to the non-dimensionalized time derivative $\mathbf{r}_{t}$ in (11), in which the surface normal is evaluated at the initial instant. This furnishes the approximation $u=\mathbf{n} \cdot \mathbf{r}_{t}$ to the normal velocity at time $\Delta \bar{t}$ which is used to integrate (62) for the tangential velocity field at time $\Delta \bar{t}$, assuming the tangential velocity at $\bar{a}=0$ to vanish and with the mean curvature equal to its initial distribution. This information is then used with (64) to evaluate the time derivative of the protein density at time $\Delta \bar{t}$, and the protein density at time $\Delta \bar{t}$ is again obtained by forward integration. The procedure is repeated for a specified time interval or until an equilibrium state is reached, the latter being identified with the limit of a sequence of states computed at a succession of time steps.

\section{Examples}

In the first example, we assign the value zero to $\bar{r}, \psi$ and $\bar{L}$ at $\bar{a}=0$. These correspond, respectively, to vanishing radius, angle, and transverse shear force at the pole of the membrane. The last of these implies that there is no concentrated force at the pole [17]. The membrane is assumed to have total dimensionless area equal to $\pi(R=1)$ and we suppose that $\bar{z}(\pi)=0$. We further impose vanishing bending moment at $\bar{a}=\pi$, this being equivalent to putting $\bar{H}(\pi)=\bar{C}(\pi)$ [17]. Finally, we suppose that the radial traction $\bar{F}_{r}$ vanishes at $\bar{a}=\pi$, where

$$
F_{r}=F_{\nu} \cos \psi-F_{n} \sin \psi,
$$

in which $F_{\nu}$ is the traction in the direction of the exterior unit normal $\boldsymbol{\nu}$ to the surface edge and $F_{n}$ is the traction in the direction of $\mathbf{n}$. Explicit formulae for these components are given in [17]. They may be combined with the formula (44) for the strain energy and substituted into (68) to obtain the boundary condition

$$
\left[(\bar{\alpha} \bar{\sigma}-\bar{\beta})^{2}+\bar{\lambda}\right] \cos \psi+(\bar{L} / \bar{r}) \sin \psi=0 \quad \text { at } \quad \bar{a}=\pi
$$

Regarding the protein density and flux, we assume that the initial value of $\bar{\sigma}$ vanishes at every mesh point and that there is no protein flux $(m=0)$ at the outer edge $\bar{a}=\pi$. Finally, we impose $\bar{\sigma}=1$ at 

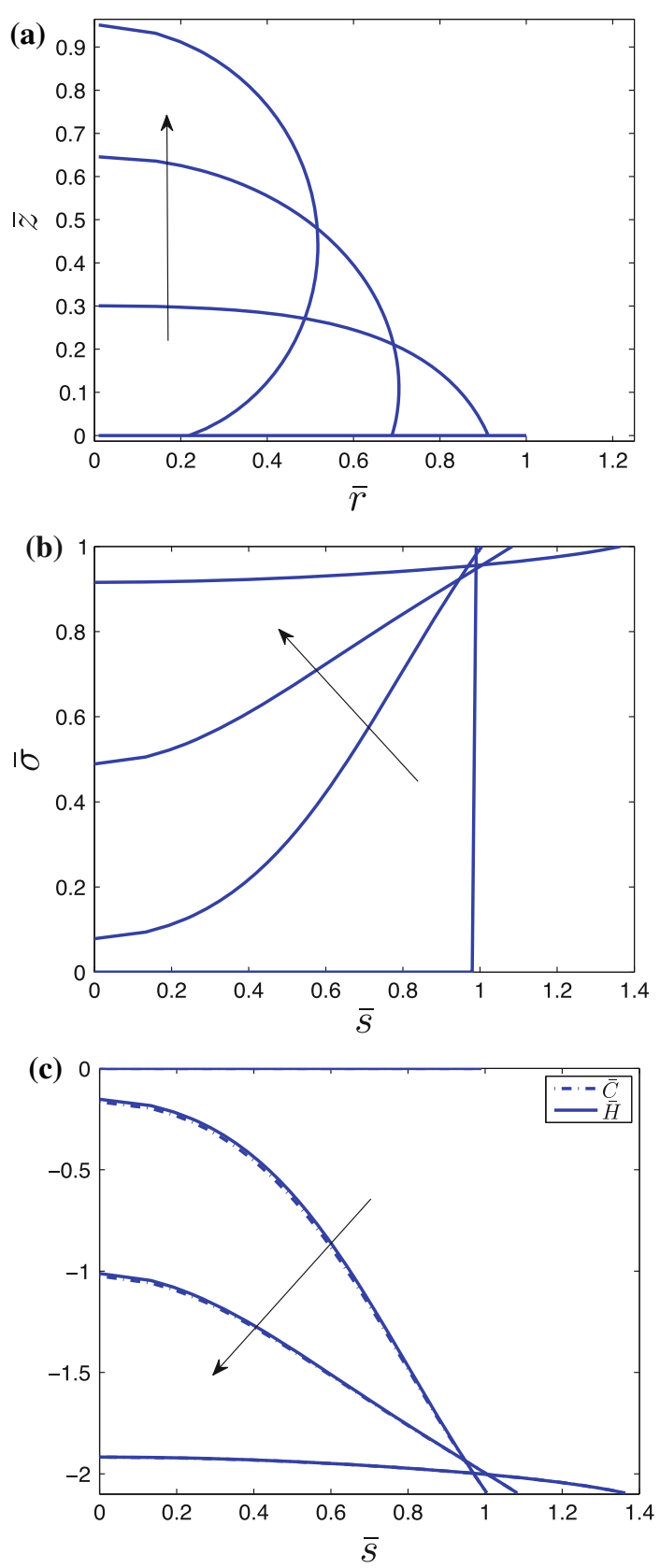

FIG. 1. a Equilibrium shapes of a membrane patch as the proteins diffuse into the domain from the outer reservoir, b protein concentration in the membrane patch, and $\mathbf{c}$ induced spontaneous curvature and mean curvature associated with the equilibrium configurations. Arrows indicate the direction of increasing time

$\bar{a}=\pi$, at the initial instant and at all subsequent times. This corresponds to the sudden exposure of the membrane to a protein bath. The problem is solved with the constants

$$
\bar{\mu} \phi=-\pi, \quad \hat{\tau}(=k c)=1, \quad \bar{\alpha}=1, \quad \bar{\beta}=0.5 \quad \text { and } \quad \Delta \bar{t}=0.001,
$$



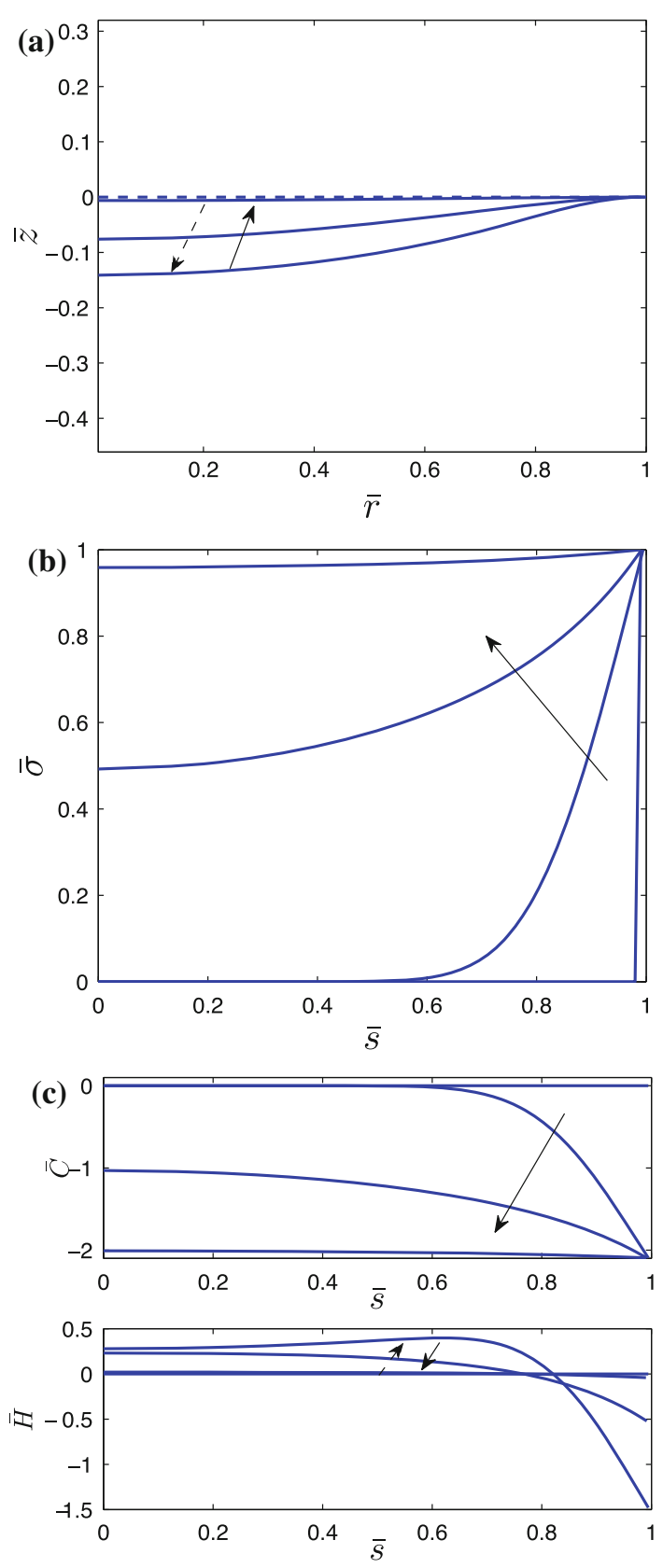

FIG. 2. a Equilibrium shapes of a membrane patch as the proteins flow in from the outer reservoir. Dashed curve corresponds to initial shape and the dashed arrow indicates the shape at the following time step. Solid arrow indicates the direction of evolution at the subsequent time steps. b Protein concentration in the membrane patch, and c induced spontaneous curvature and mean curvature associated with the equilibrium configurations. Arrows indicate the direction of increasing time

the first of which tends to promote a negative curvature of the membrane due to the cone-shaped proteins. This is illustrated in Fig. 1a, which depicts the membrane shape at a succession of times, starting with a planar configuration. The sequence of shapes is seen to become ever more curved as time advances, 

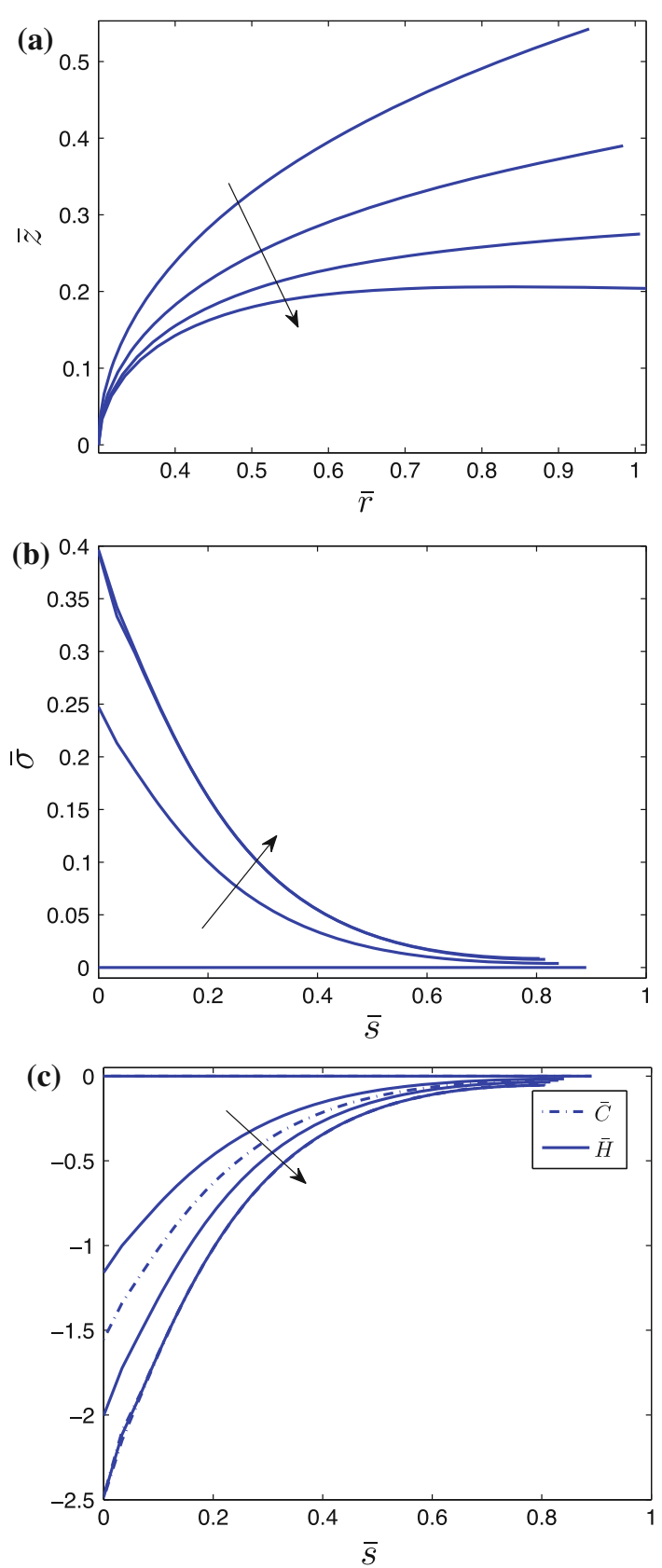

FIG. 3. a Equilibrium shapes of a membrane annulus as the proteins enter the domain from the equatorial plane, $\mathbf{b}$ protein concentration in the membrane patch, and $\mathbf{c}$ induced spontaneous curvature and mean curvature associated with the equilibrium configurations. Arrows indicate the direction of increasing time

due to the gradual increase in protein density, depicted in Fig. 1b, over the domain. In particular, we do not achieve a static configuration in this example and thus conclude, in agreement with [3], that protein diffusion has the potential to destabilize the planar membrane shape. The mean and spontaneous curvatures are seen, in Fig. 1c, to be nearly equal in all states of the system, implying that the process is driven primarily by conventional Fickean diffusion. 
The data for the second example differ from those for the first only in the replacement of the zero-moment condition with the clamping condition $\psi=0$ at $\bar{a}=\pi$. The tendency of the proteins to induce a negative curvature manifests itself near the edge of the membrane, where the protein concentration is greatest. However, the curvature must change sign in the interior as the axis of symmetry is approached because of continuity with the interior part of the membrane where protein density is initially low and consequently where the membrane is nearly flat. As the protein density in the interior increases due to diffusion, there is a tendency for the curvature there to rebound to negative values, but this is hindered by the higher concentration of proteins near the edge, which act to maintain a localized negative curvature. This effect combines with the clamping condition to produce a sequence of shapes that initially descends below the plane $\bar{z}=0$, followed by a reversal of the sign of the normal velocity, leading the membrane back to a planar configuration as time advances. The associated equilibrium distribution of proteins is non-uniform. These effects are depicted in Fig. 2a-c.

The third and final example pertains to the geometry of a nuclear pore [8]. The protein concentration is again assumed to vanish in the interior, at the initial instant. Thereafter, a flux is assigned at $\bar{a}=0$. This is taken to be linear in time, with a positive coefficient, corresponding to a net influx of proteins at the inner boundary in accordance with the definition of the edge normal $\boldsymbol{\nu}$ above, which is directed into the part of the membrane above the equator. This flux is subsequently set to zero after an interval of time, so that thereafter no additional proteins enter the domain. Zero flux is assigned at the outer boundary $(\bar{a}=\pi)$. We assign vanishing normal force (i.e., $\bar{L}=0)$ at the inner and outer boundaries, and assume the bending moment to vanish $(\bar{H}=\bar{C})$ at the outer boundary. The condition on force at the inner boundary is necessary if the solution is to possess reflection symmetry with respect to the equator [24]. Finally, we impose the geometric conditions $\bar{z}=0, \bar{r}=0.3$, and $\psi=\pi / 2$ at the inner boundary. These pertain to a pore of finite radius where the membrane cuts the equatorial plane vertically. The solution is obtained for the values

$$
\bar{\mu} \phi=-2 \pi, \quad \hat{\tau}(=k c)=1, \quad \bar{\alpha}=0, \quad \bar{\beta}=0 \quad \text { and } \quad \Delta \bar{t}=0.001,
$$

corresponding to the bending-dominated regime described by Eq. (49).

The initial equilibrium shape is found to be a catenoid $(\bar{H}=0)$. As time advances and more proteins diffuse into the interior, the curvature of the region near the inner boundary, close to the axis of symmetry, increases sharply due to the strong local accumulation of proteins (Fig. 3a). This concentration diminishes rapidly away from the inner boundary, resulting in a gradual transition to a region with nearly zero curvature (Fig. 3b,c). The simulation furnishes an equilibrium shape very much like those observed in the immediate vicinity of a nuclear pore where protein density is greatest [8], thus supporting the view that pore morphology is mediated by a surface distribution of transmembrane proteins.

\section{References}

1. Ou-Yang, Z.-C., Liu, J.-X., Xie, Y.-Z.: Geometric Methods in the Elastic Theory of Membranes in Liquid Crystal Phases. World Scientific, Singapore (1999)

2. Kim, K.S., Neu, J., Oster, G.: Curvature-mediated interactions between membrane proteins. Biophys. J. 75, 22742291 (1998)

3. Leibler, S.: Curvature instability in membranes. J. Phys. 47, 507-516 (1986)

4. Reister, E., Seifert, U.: Lateral diffusion of a protein on a fluctuating membrane. Europhys. Lett. 71, 859-865 (2005)

5. Seifert, U.: Configurations of fluid membranes. Adv. Phys. 46, 13-137 (1997)

6. Arroyo, M., DeSimone, A.: Relaxation dynamics of fluid membranes. Phys. Rev. E Stat. Nonlin. Soft Matter Phys. 79, 031915 (2009)

7. Góźdź, W.T.: Shape transformations of lipid vescicles induced by diffusing macromolecules. J. Chem. Phys. 134, 024110-1-024110-8 (2011)

8. Agrawal, A., Steigmann, D.J.: Modeling protein-mediated morphology in biomembranes. Biomech. Model. Mechanobiol. 8, 371-379 (2009)

9. Helfrich, W.: Elastic properties of lipid bilayers: theory and possible experiments. Z. Naturforsch. 28, 693-703 (1973) 
10. Jenkins, J.T.: The equations of mechanical equilibrium of a model membrane. SIAM J. Appl. Math. 32, 755-764 (1977)

11. Steigmann, D.J.: Fluid films with curvature elasticity. Arch. Rational Mech. Anal. 150, 127-152 (1999)

12. Aris, R.: Vectors, Tensors and the Basic Equations of Fluid Mechanics. Dover, New York (1989)

13. Kreyzsig, E.: Differential Geometry. University of Toronto Press, Toronto (1959)

14. Sokolnikov, I.S.: Tensor Analysis: Theory and Applications to Geometry and Mechanics of Continua. Wiley, New York (1964)

15. Naghdi, P.M.: Theory of shells and plates. In: Truesdell, C. (ed.) Handbuch der Physik, vol. VIa/2, pp. 425-640. Springer, Berlin (1972)

16. Scriven, L.E.: Dynamics of a fluid interface. Chem. Eng. Sci. 12, 98-108 (1960)

17. Agrawal, A., Steigmann, D.J.: Boundary-value problems in the theory of lipid membranes. Continuum Mech. Thermodyn. 21, 57-82 (2009)

18. Steigmann, D.J., Baesu, E., Rudd, R.E., Belak, J., McElfresh, M.: On the variational theory of cell-membrane equilibria. Interfaces Free Boundaries 5, 357-366 (2003)

19. Gurtin, M.E., Fried, E., Anand, L.: The Mechanics and Thermodynamics of Continua. Cambridge University Press, New York (2010)

20. Steigmann, D.J., Li, D.: A theory of elasticity for fluid films. Proc. R. Soc. Lond. A 449, 223-231 (1995)

21. Steigmann, D.J., Li, D.: Energy minimizing states of capillary systems with bulk, surface and line phases. IMA J. Appl. Math. 55, 1-17 (1995)

22. Evans, E.A., Skalak, R.: Mechanics and Thermodynamics of Biomembranes. CRC Press, Boca Raton (1980)

23. Pipkin, A.C., Rivlin, R.S.: The formulation of constitutive equations in continuum physics. Technical Report No. 4, Department of the U.S. Army, Project No. 599-01-004 (DA-4531/4) (1958)

24. Agrawal, A., Steigmann, D.J.: Coexistent fluid-phase equilibria in biomembranes with bending elasticity. J. Elasticity 93, 63-80 (2008)

Ashutosh Agrawal

Department of Applied Physics

California Institute of Technology

Pasadena

CA 91125

USA

David J. Steigmann

Department of Mechanical Engineering

University of California

Berkeley

CA 94720

USA

e-mail: steigman@newton.berkeley.edu

(Received: November 1, 2010; revised: April 18, 2011) 